\title{
Suppression of Sirtuin-1 Increases IL-6 Expression by Activation of the Akt Pathway During Allergic Asthma
}

\author{
Lingling Tang ${ }^{a}$ Qingge Chen ${ }^{a}$ Ziyu Meng ${ }^{a} \quad$ Li Sun $^{a} \quad$ Linyun Zhu ${ }^{a} \quad$ Jinjin Liu ${ }^{a}$ \\ Junsheng $\mathrm{Hu}^{\mathrm{a}}$ Zhenhua $\mathrm{Ni}^{\mathrm{b}}$ Xiongbiao Wang ${ }^{\mathrm{a}}$ \\ aDepartment of Respiratory Medicine, Putuo Hospital, Shanghai University of Traditional Chinese \\ Medicine, Shanghai, ${ }^{b}$ Central lab, Putuo Hospital, Shanghai University of Traditional Chinese Medicine, \\ Shanghai, China
}

\section{Key Words}

Asthma $\cdot$ Sitruin-1 $\cdot$ Interleukin-6

\begin{abstract}
Background/Aims: A growing number of studies have demonstrated that the activity and expression level of sirtuin-1 (SIRT1) are decreased in asthma patients; however, the mechanisms underlying decreased SIRT1 expression and function are still not completely understood. Interleukin (IL)- 6 plays important roles in inflammation during allergic asthma. In this study, we examined whether loss of SIRT1 activity regulated the expression of IL- 6 and further verified the underlying mechanisms. Methods: The human airway epithelial cell line $16 \mathrm{HBE}$ was used to test the effects of the SIRT1 inhibitor (salermide) on expression of IL-6. IL-6 mRNA and protein expression were assessed with real-time polymerase chain reaction $(P C R)$, immunochemistry, and ELISA. OVA-challenged mice were used as an asthma model to investigate the effect of SIRT1 activation on IL-6 and relative Akt phosphorylation level. Results: We found that inhibition of SIRT1 increased IL- 6 mRNA and protein levels in a timedependent manner, which was accompanied by increased Akt pathway activation in $16 \mathrm{HBE}$ cells. Furthermore activation of Akt showed upregulated expression of the IL- 6 protein whereas Akt inhibitor, LY294002 or Akt siRNA significantly inhibited SIRT1-regulated IL-6 expression. Conversely, activation of SIRT1 inhibited Akt activation and IL- 6 expression in an asthmatic mice model and 16HBE cells. Conclusion: Our results indicate the potential role of SIRT1 in regulating inflammation by modulation of IL- 6 expression in an Akt-dependent manner during allergic asthma.

Zhenhua $\mathrm{Ni}_{\text {, }}$

and Xiongbiao Wang

KARGER
Central lab, Putuo Hospital, Shanghai University of Traditional Chinese Medicine

Shanghai, and Department of Respiratory Medicine, Putuo Hospital, Shanghai

University of Traditional Chinese Medicine, Shanghai, (China)

E-Mail zhenhuani@yeah.net, xiongbiao6@yahoo.com 


\section{Cellular Physiology Cell Physiol Biochem 2017;43:1950-1960

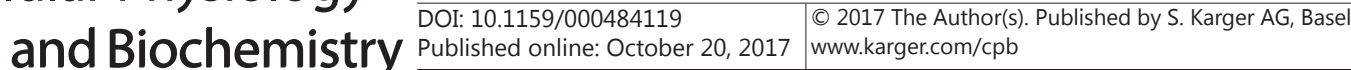 \\ Tang et al.: SIRT1 Increases IL-6 Expression by Activating Akt}

\section{Introduction}

Asthma is one of the most common chronic immunological diseases that involves a complex interplay of environmental factors [1]. The development of a CD4 Th2 immune response and the associated cytokines are known to play an important role in the pathogenesis of asthma. Therefore, Th2 cytokine inhibition is a significant potential drug target for future asthma treatment strategies.

Sirtuin 1 (SIRT1), also known as NAD-dependent deacetylase sirtuin-1, is a member of the sirtuin family of proteins. SIRT1 has been shown to mediate a variety of cellular events, including life-span extension, cell growth, and inflammation [2-4]. Since asthma is an inflammatory disease, the roles of SIRT1 in the physiological and pathological process of asthma have been investigated by several studies. In OVA-sensitized and challenged mice, Ichikawa et al. found that the expression of SIRT1 mRNA in the lungs was decreased when compared with that in control mice [5]. Moreover, they found that treatment of OVA mice with a SIRT1 activator decreased inflammatory cell lung infiltrates and IL-5 and IL13 levels in the BAL fluid compared to those in the vehicle treatment [6] , which indicated the anti-inflammation effects of SIRT1. In addition, Wang et al. also confirmed decreased SIRT1 expression in the lung tissues of OVA mice [7]. In asthma patients, Colley et al. found SIRT1 activity was decreased in PBMCs from patients with severe asthma when compared to healthy subjects, and loss of SIRT1 activity increases IL-4 expression in patients with severe asthma [8]. Therefore, SIRT1 might act as an important pro-inflammatory mediator during allergic asthma.

Interleukin (IL)-6 is a Th2 cytokine that is now emerging as a potentially pivotal player in the regulation of inflammation. Clinical studies have shown that IL-6 is consistently elevated in the airways of children and adults with asthma, and high IL-6 levels may be an indication of forced expiratory volume in 1 second (FEV1) and forced vital capacity (FVC) $[9,10]$. IL-6 has recently emerged as a main regulator of the differentiation and function of Th17 cells $[11,12]$. In addition, Lin et al. reported that IL-6 signalling was important for DCs to take up allergens and to initiate Th2/Th17-mediated airway inflammation and AHR in asthma [13]. Furthermore, they found DCs from allergic asthmatics treated with anti-IL-6 receptor antibody had poor capacities for eliciting Th2 polarization, which indicated that IL-6 plays an important role in regulating disease severity.

Therefore, in the present study, we examined whether loss of SIRT1 activity modulated the expression of IL- 6 and further verified the mechanisms by which SIRT1 regulated IL-6 expression.

\section{Materials and Methods}

\section{Animals}

All experimental procedures complied with the international standards of animal welfare and were approved by the Institutional Animal Care and Use Committee of Shanghai University of Traditional Chinese Medicine. Female six-to-eight-week BALB/c mice were purchased from SLRC Laboratory Animal (Shanghai, China). All mice were kept in well-controlled animal housing facilities and had free access to tap water and food pellets throughout the experimental period.

Ovalbumin (OVA)-induced mice model of asthma, and resveratrol treatment.

Six-to-eight-week old BALB/c mice $(n=15)$ were divided into the following three groups: a saline group (saline-induced mice treated with saline, $n=5$ ), an OVA group (OVA-induced mice treated with DMSO, $n=5$ ), an OVA/Res group (OVA-induced mice treated with resveratrol, $n=5$ ). All OVA induced mice were immunized via intraperitoneal (i.p.) injections of $100 \mu \mathrm{g}$ of OVA (Grade V; Sigma, St. Louis, MO, U.S.A.) complexed with alum on days 0 and 14. Then mice from OVA group and OVA/Res group received an intranasal dose of $50 \mu \mathrm{L}$ OVA $(100 \mu \mathrm{g})$ and intraperitoneal injections of resveratrol (30 mg/kg, sigma) on days 14, 25, 26, and 27. Between days 28 and 34, the resveratrol group was injected with $30 \mathrm{mg} / \mathrm{kg}$ resveratrol (i.p.) once a day; 


\section{Cellular Physiology Cell Physiol Biochem 2017;43:1950-1960 \begin{tabular}{l|l|l} 
and Biochemistry Published online: October 20, 2017 & $\begin{array}{l}\text { (c) } 2017 \text { The Author(s). Published by S. Karger AG, Basel } \\
\text { www.karger.com/cpb }\end{array}$
\end{tabular} \\ Tang et al.: SIRT1 Increases IL-6 Expression by Activating Akt}

the control and asthma groups received saline. On day 35, $24 \mathrm{~h}$ following each experimental group's final treatment, the mice were sacrificed, and the lungs and serum were dissected out for further analysis.

Cell culture

Human bronchial epithelial cells (16HBE) were maintained in RPMI medium 1640 (Thermo Fisher Scientific, Inc., Waltham, MA, USA) supplemented with 10\% fetal bovine serum, penicillin, and streptomycin.

Gene silencing

Small-interfering RNAs targeting human Akt (Gene ID: 207) were synthesized (GenePharma, Shanghai, China) and transfected into cells by using TransIT-TKO (Mirus, Madison, WI, USA). Scrambled siRNA was used as a control. The sequences of siRNA used for Akt silencing were shown below: Akt siRNA, 5'GACGGGCACATTAAGATCA-3'; control siRNA, 5'TTCTCCGAACGTGTCATGT-3'.

Gene silencing of SIRT1

Lenti virus-containing short hairpin RNA (shRNA) interference against the human SIRT1 gene (Gene ID: 23411) was designed and constructed by Genechem (Genechem, Shanghai, China). The sequence was listed below:5-TCGAACAATTCTTAAAGAT-3. A control shRNA (5-TTCTCCGAACGTGTCACGT-3) was used as a negative control. The 16HBE cells were infected with SIRT1 lentivirus at an MOI of approximately 100. After $72 \mathrm{~h}$ of infection, cells were harvested for further study

\section{Real-time quantitative PCR( $(P C R)$}

Total RNA was isolated from the lungs using Trizol reagent (TaKaRa, Dalian, China), and firststrand cDNAs were prepared using a random hexamer primer according to the instructions included with the First-Strand Synthesis Kit (Roche, San Francisco, USA). Polymerase chain reactions were performed using specific forward and reverse primers(hIL-6, forward, 5'-CCTGAACCTTCCAAAGATGGC-3', reverse,5'-TTCACCAGGCAAGTCTCCTCA-3'; hß-actin, forward, 5'-CCAACCGCGAGAAGATGA-3', reverse, 5'-CCAGAGGCGTACAGGGATAG-3'; mIL-6, forward, 5'- TCTATACCACTTCACAAGTCGGA-3', reverse, 5'-GAATTGCCATTGCACAACTCTTT-3'; mSIRT1, forward, 5'-CAGCCGTCTCTGTGTCACAAA-3', reverse, 5'GCACCGAGGAACTACCTGAT--3'; m 3 -actin, forward, 5'-GGCTGTATTCCCCTCCATCG-3', reverse, 5'-CCAGTTGGTAACAATGCCATGT-3'). Real-time PCR was performed using Universal Master Mixer (Roche). The relative expression levels of the IL- 6 gene was normalized against $\beta$-actin and analyzed via the 2- $\Delta \Delta \mathrm{Ct}$ method $\left[\Delta \Delta \mathrm{Ct}=\left(\mathrm{Ct}{ }_{\text {Target }}-\mathrm{Ct} \mathrm{Reference}\right)\right.$ sample $-\left(\mathrm{Ct}{ }_{\text {Target }}-\mathrm{Ct}{ }_{\text {Reference }}\right)$ control $]$.

Enzyme-linked immunosorbent assay of IL-6 protein level

The protein from intracellular was extracted by cell lysis reagent (Cell Signaling Technology, Danvers, USA). The supernatants were harvest after centrifuge $12000 \mathrm{rpm}$ for $15 \mathrm{~min}$ in $4^{\circ} \mathrm{C}$. The total protein concentration was determined by BCA protein assay. A total of $20 \mu \mathrm{g}$ protein was used to measure the IL- 6 levels in the protein from intracellular using the human IL-6 Quantikine ELISA kit (BD Biosciences, San Jose, $\mathrm{CA}$ ), according to the manufacturer's instructions. Absorbance at $450 \mathrm{~nm}$ was read by a microplate reader.

Western blot

The cells were washed once in phosphate-buffered saline (PBS) and dissolved in cell lysis reagent (Cell Signaling Technology, Danvers, USA). The supernatants were harvest after centrifuge 12000rpm for $15 \mathrm{~min}$ in $4^{\circ} \mathrm{C}$. The total protein was separated on a $10 \%$ SDS-PAGE, followed by transferring to PVDF membrane. The PVDF membrane was blocked with 5\% BSA, washed twice with TBST, and then incubated with the antibodies separately overnight at $4^{\circ} \mathrm{C}$. The antibodies to $\beta$-actin, Akt, P-Akt-Thr308, mTOR,

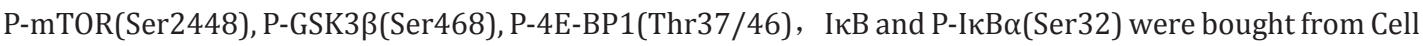
Signaling (Cell Signaling Technology) and the antibodies to P-Akt-Ser473 were bought from Abcam (Abcam, Cambridge, UK). The membrane was then washed with TBST three times followed by incubation with antirabbit IgG or anti-mouse IgG horseradish peroxidase secondary antibody (Cell Signaling Technology) for $2 \mathrm{~h}$ at room temperature. Finally, immunoreactive bands were detected by ECL reagent.

\section{Immunohistochemistry}

Immunohistochemistry was conducted as described previously [14]. Tissue sections from the left lung of the mice were deparaffinized in xylene, and rehydrated in graded ethanol. Sections were boiled at a constant temperature of $95^{\circ} \mathrm{C}$ for $15 \mathrm{~min}$ in citrate buffer $(0.01 \mathrm{mmol} / \mathrm{l}$; pH 6.0; Fuzhou Maixin Biotechnology 


\section{Cellular Physiology Cell Physiol Biochem 2017;43:1950-1960 \begin{tabular}{l|l|l} 
DOI: 10.1159/000484119 & $\begin{array}{l}\text { O 2017 The Author(s). Published by S. Karger AG, Basel } \\
\text { www.karger.com/cpb }\end{array}$
\end{tabular} \\ Tang et al.: SIRT1 Increases IL-6 Expression by Activating Akt}

Development Co, Fuzhou, China) for antigen retrieval. The tissue sections were first incubated with mIL-6 monoclonal antibody (Santa Cruz Biotechnology, Dallas, TX, USA) and P-Akt-Ser473 (Abcam) overnight at $4^{\circ} \mathrm{C}$. Following incubation, tissue sections were then washed with phosphate buffered saline (PBS). and treated with a streptavidin-biotin-peroxidase complex (SABC kit, Boster, Wuhan, China). Signal detection was performed using diaminobenzidine (DAB). IL-6 and P-Akt-Ser473 staining intensity was analyzed by measuring integrated optimal density (IOD) values, using Image-Pro Plus 6.0.

\section{Assessment of SIRT1 activity}

Cyclex SIRT1/Sir2 Deacetylase Fluorometric Assay Kit (MBL life science, Japan) was used to measure SIRT1 activity according to the manufacturer's protocol. The total protein from intracellular was mixed with the reagents of SIRT1/Sir2 Deacetylase Fluorometric Assay Kit and incubated for 60 min at room temperature. The fluorescence intensity was then measured in a microplate fluorescence reader with excitation at $355 \mathrm{~nm}$ and emission at $460 \mathrm{~nm}$. The results are reported as fluorescence/ $\mu \mathrm{g}$ of protein.

\section{Statistics}

SPSS version 21.0 software was used for data analyses. Data are expressed as the mean \pm standard deviation. Statistical significance was determined by Student's t-test, linear correlation, or Mann-Whitney U test. Statistically significant differences were defined as $\mathrm{P}<0.05$.

\section{Results}

\section{Suppression of SIRT1 increases IL-6 production in human lung epithelial cells}

To investigate whether SIRT1 could inhibit IL-6 expression in human airway cells, 16HBE human lung epithelial cells were treated with the SIRT1 inhibitor salermide for different times. As previous studies demonstrated, the increased acetylation status of p53 is considered a marker of decreased SIRT1 activity in vitro [15]. We found that salermide treatment decreased SIRT1 activity and increased acetylation status of p53 (Fig. 1A-C).

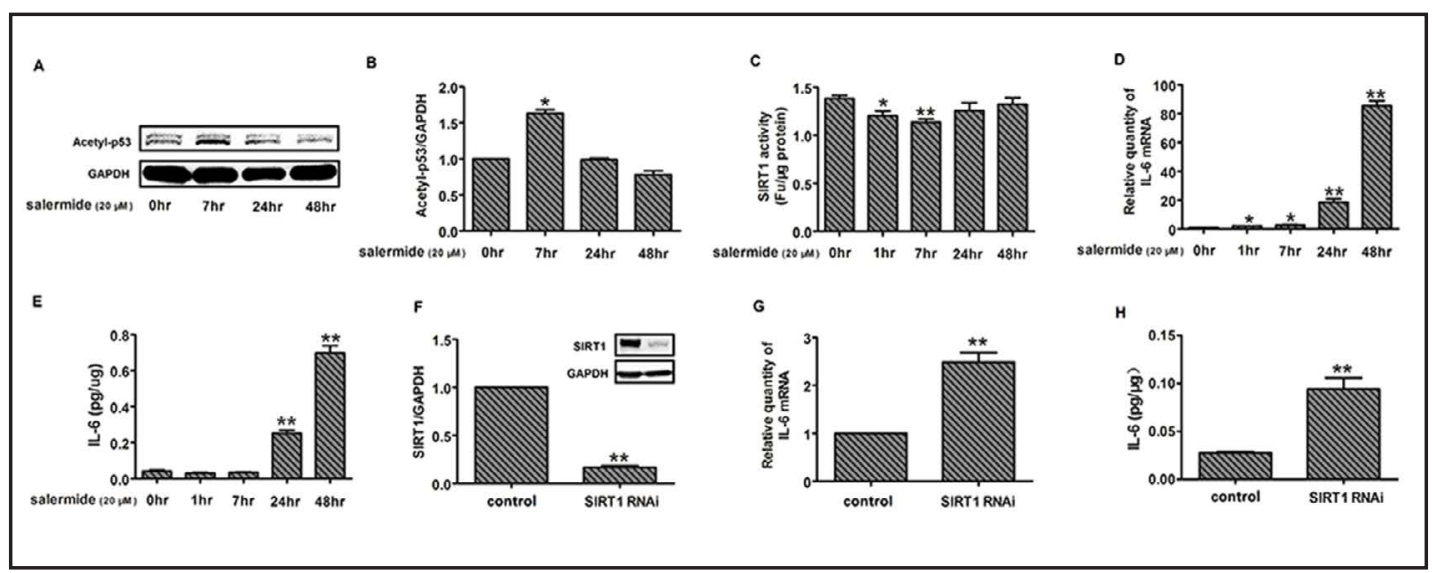

Fig. 1. Suppression of SIRT1 increases IL-6 production in human lung epithelial cells. Cultured 16HBE cells were treated with salermide $(20 \mu \mathrm{M})$ for different time $(0 \mathrm{~h}, 7 \mathrm{~h}, 24 \mathrm{~h}$ and $48 \mathrm{~h})$. Acetyl-p53 were measured by using western blotting $(A, B)$. Acetyl-p53 protein concentrations were quantified by using Image J software and normalized to GAPDH. SIRT1 activity was measured by Cyclex SIRT1/Sir2 Deacetylase Fluorometric Assay Kit (C). Cultured $16 \mathrm{HBE}$ were treated with salermide $(20 \mu \mathrm{M})$ for different time $(0 \mathrm{~h}, 1 \mathrm{~h}, 7 \mathrm{~h}, 24 \mathrm{~h}$ and $48 \mathrm{~h})$. The mRNA and protein from intracellular expression of IL- 6 was determined via qPCR (D) and ELISA (E) respectively. The SIRT1 RNAi and control RNAi lentivirus were transfected into 16HBE cells, and the protein levels of SIRT1 were analyzed by using western blotting (F). SIRT1 protein concentrations were quantified by using Image J software and normalized to GAPDH. The mRNA and protein from intracellular expression of IL-6 in SIRT1 knockdown cells and control cells were determined via qPCR (G) and ELISA (H) respectively. Data are presented as mean $\pm \mathrm{SEM}$ from three independent, $* \mathrm{P}<0.05, \quad * * \mathrm{P}<0.01$. 


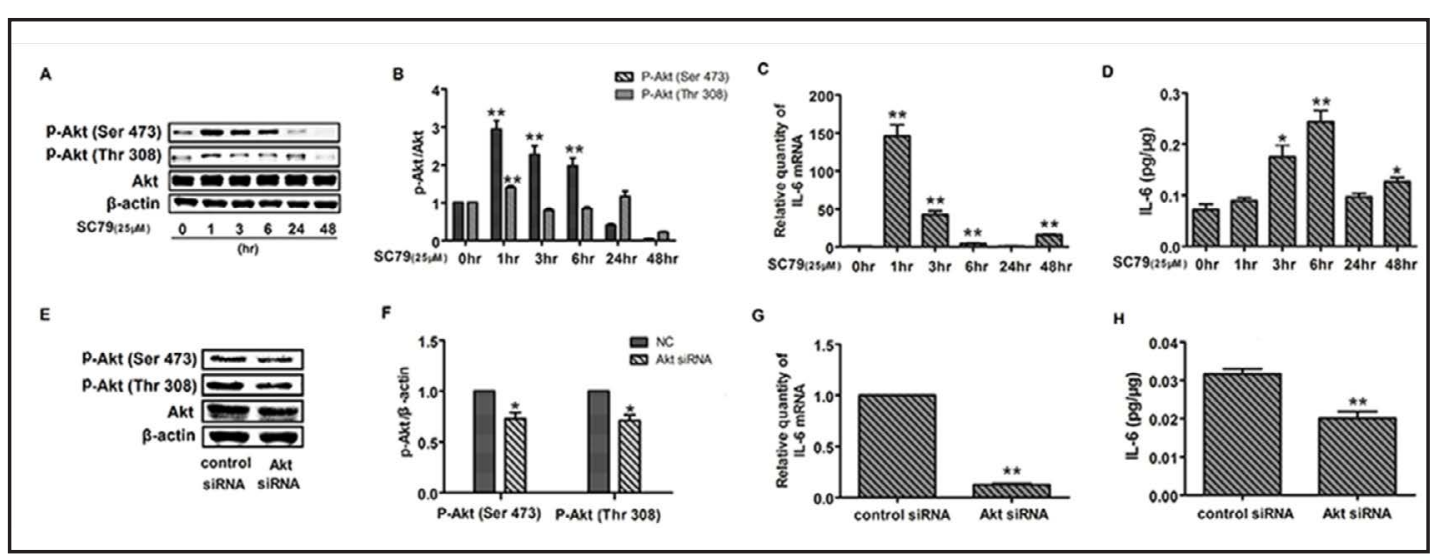

Fig. 2. Akt activation increased IL-6 expression in human lung epithelial cells. 16HBE cells were treated with SC79 $(25 \mu \mathrm{M})$ for different time $(0 \mathrm{~h}, 1 \mathrm{~h}, 3 \mathrm{~h}, 6 \mathrm{~h}, 24 \mathrm{~h}$ and $48 \mathrm{~h})$. The levels of the relative Akt phosphorylation at Ser473 and Thr308, and total Akt were measured by using western blotting (A). Phosphoprotein concentrations were quantified by using Image J software and normalized to total Akt (B). The expression of IL-6 mRNA determined via qPCR (C), and protein from intracellular (D) determined via ELISA. The siRNA duplexes against Akt were transfected into 16HBE cells, and the relative Akt phosphorylation at Ser473 and Thr308 and total Akt were analyzed by using western blotting at $24 \mathrm{~h}$ after the siRNA transfections (E). Protein concentrations were quantified by using Image J software and normalized to $\beta$-actin (F). The mRNA and protein from intracellular expression of IL- 6 was determined via qPCR (G) and ELISA (H) respectively. Data are presented as mean $\pm \mathrm{SEM}$ from three independent, $* \mathrm{P}<0.05, \quad{ }^{*} \mathrm{P}<0.01$.

We also found inhibition of SIRT1 activity by salermide increased IL-6 mRNA and protein expression in a time-dependent manner (Fig. 1D, E). These results indicated loss of SIRT1 activity increases IL- 6 production. Furthermore, we used RNA interference to decrease SIRT1 expression (Fig. 1F). Our results showed SIRT1 knockdown increased IL-6 expression levels, which confirmed the role of SIRT1 in IL-6 regulation (Fig. 1G, H).

Akt activation increased IL-6 expression in human lung epithelial cells

It was reported that the PI3K/Akt pathway might play important roles in modulating IL-6 release in various cells $[16,17]$. Thus, we examined whether Akt activation increased IL-6 expression in human lung epithelial cells. Using the Akt activator SC79, a unique and specific Akt activator that has been used to enhance Akt activity in various physiological and pathological conditions [18]. We found that SC79-stimulated 16HBE cells displayed increased relative Akt phosphorylation at Ser473 and Thr308 (Fig. 2A, B) and IL-6 mRNA (Fig. 2C) and protein from intracellular (Fig. 2D). To further investigate the effects of Akt activation on IL-6 expression, we inhibited Akt expression using RNA interference. As shown in Fig. 2E, F, Akt protein, and relative Akt phosphorylation at Ser473 and Thr308 were reduced by Akt siRNA treatment, and the levels of IL- 6 mRNA and protein were also decreased in Akt siRNA-treated cells than in control cells (Fig. 2G, H). These results indicated Akt activation increased IL-6 expression in human lung epithelial cells.

\section{Suppression of SIRT1 increases Akt activation in human lung epithelial cells}

Based on these results, we next examined the effects of SIRT1 on Akt activation. After treatment with the SIRT1 inhibitor salermide, we observed an increase in relative Akt phosphorylation at Ser473 and Thr308, relative mTOR phosphorylation at Ser2448, relative GSK3 $\beta$ phosphorylation at Ser468, relative 4E-BP1 phosphorylation at Thr37/46, relative $\mathrm{I \kappa B} \alpha$ phosphorylation at Ser32 in 16HBE cells compared to control cells (Fig. 3A-G), which indicated suppression of SIRT1 activity increased Akt pathway activation in human lung epithelial cells. 


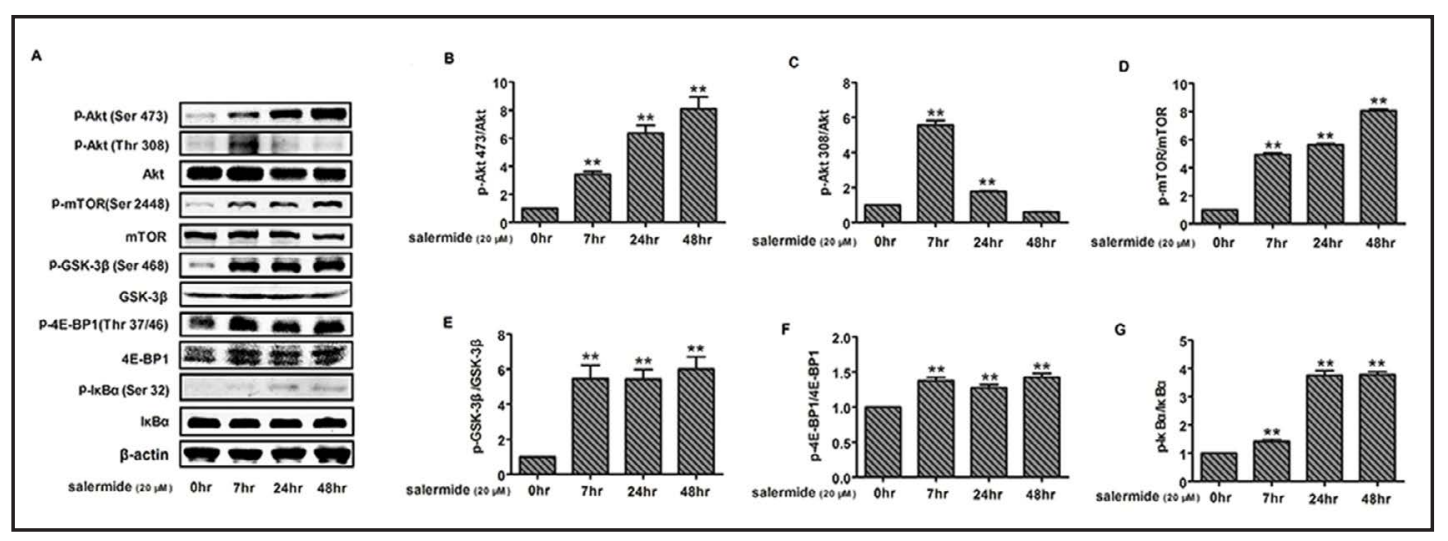

Fig. 3. Suppression of SIRT1 increases Akt activation in human lung epithelial cells. 16HBE cells were treated with salermide $(20 \mu \mathrm{M})$ for different time $(0 \mathrm{~h}, 7 \mathrm{~h}, 24 \mathrm{~h}$ and $48 \mathrm{~h})$. The levels of relative Akt phosphorylation at Ser473 and Thr308, total Akt, relative mTOR phosphorylation at Ser2448, total mTOR, relative GSK3 $\beta$ phosphorylation at Ser468, total GSK3 $\beta$, relative 4E-BP1 phosphorylation at Thr37/46, total 4E-BP, relative $\mathrm{I} \kappa \mathrm{B} \alpha$ phosphorylation at Ser32 and $\mathrm{I} \kappa \mathrm{B} \alpha$ and total $\mathrm{I} \kappa \mathrm{B} \alpha$ were measured by western blotting (A). Phosphoprotein concentrations were quantified by using Image J software and normalized to total protein respectively (B, C, D, E, F, G). Data are presented as mean \pm SEM from three independent, ${ }^{*} \mathrm{P}<0.05,{ }^{* *} \mathrm{P}<0.01$.

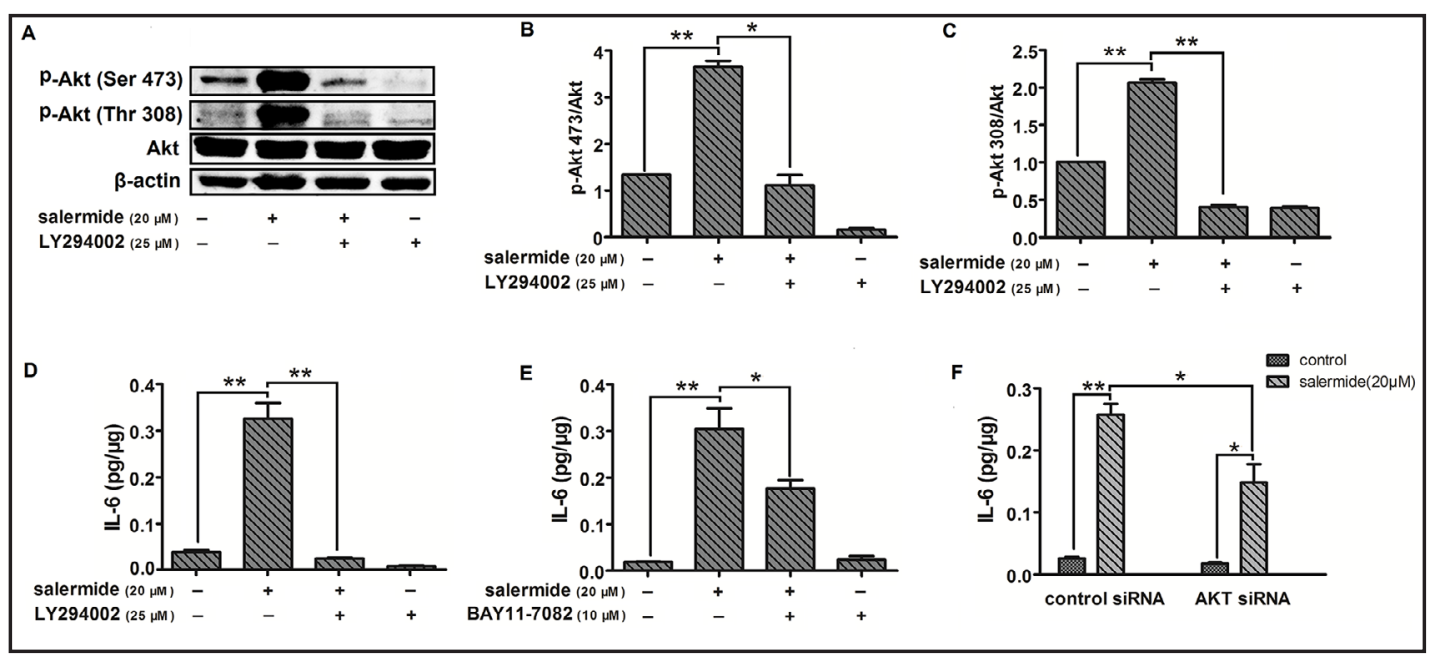

Fig. 4. Akt pathways mediated SIRT1 inhibition-induced IL-6 expression. 16HBE cells were treated with salermide $(20 \mu \mathrm{M})$, LY294002 $(25 \mu \mathrm{M})$, or salermide $(20 \mu \mathrm{M})$ plus LY294002 $(25 \mu \mathrm{M})$ for $24 \mathrm{~h}$. The protein from intracellular level of relative Akt phosphorylation was determined via Western blot (A). Phosphoprotein concentrations were quantified by using Image J software and normalized to total Akt protein (B, C). The protein from intracellular level of IL-6 was determined via ELISA (D).16HBE cells were treated with salermide $(20 \mu \mathrm{M})$, BAY 11-7082 $(10 \mu \mathrm{m} / \mathrm{L})$, or salermide $(20 \mu \mathrm{M})$ plus BAY 11-7082 $(10 \mu \mathrm{m} / \mathrm{L})$ for $24 \mathrm{~h}$, The protein from intracellular expression of IL- 6 were measured by using ELISA (E). 16HBE were transfected by siRNA for $24 \mathrm{hr}$ and then incubated in the presence of salermide $(20 \mu \mathrm{M})$ for $24 \mathrm{~h}$, The protein from intracellular expression of IL- 6 were measured by using ELISA (F). Data are presented as mean \pm SEM from three independent, ${ }^{*} \mathrm{P}<0.05, \quad{ }^{*} \mathrm{P}<0.01$.

\section{Akt pathways mediated SIRT1 inhibition-induced IL-6 expression}

To further identity the molecular mechanism mediating pro-inflammatory effects of SIRT1, we examined the effects of Akt and IкB inhibitor on SIRT1 inhibition-induced IL-6 expression. As shown in Fig. 4A to D, Akt inhibitor LY294002 significantly inhibited salermide-induced relative Akt phosphorylation and IL-6 expression in 16HBE cells. IкB inhibitor BAY11-7082 significantly inhibited salermide-induced protein expression of IL-6 


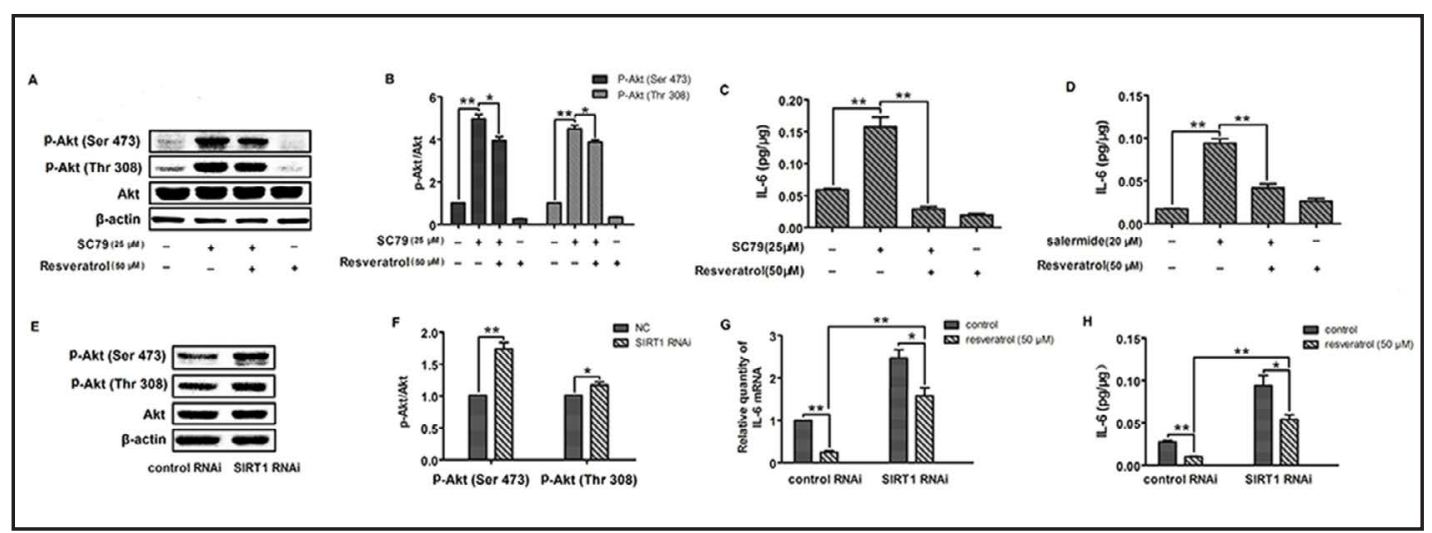

Fig. 5. SIRT1 activator inhibited IL-6 expression in human lung epithelial cells. 16HBE cells were treated with SC79 $(25 \mu \mathrm{M})$, resveratrol $(50 \mu \mathrm{M})$, or SC79 $(25 \mu \mathrm{M})$ plus resveratrol $(50 \mu \mathrm{M})$ for $24 \mathrm{~h}$. The protein from intracellular level of relative Akt phosphorylation was determined via Western blot (A). Phosphoprotein concentrations were quantified by using Image J software and normalized to total Akt protein (B). The protein from intracellular level of IL-6 was determined via ELISA (C). 16HBE cells were treated with salermide $(20 \mu \mathrm{M})$, resveratrol $(50 \mu \mathrm{M})$, or salermide $(20 \mu \mathrm{M})$ plus resveratrol $(50 \mu \mathrm{M})$ for $24 \mathrm{~h}$. The protein from intracellular expression of IL-6 were determined via ELISA (D). The SIRT1 RNAi and control RNAi lentivirus were transfected into $16 \mathrm{HBE}$ cells, and then incubated in the presence of resveratrol $(50 \mu \mathrm{M})$ for $24 \mathrm{~h}$. The protein from intracellular level of relative Akt phosphorylation was determined via Western blot (E). Phosphoprotein concentrations were quantified by using Image J software and normalized to total Akt protein (F). The mRNA and protein from intracellular expression of IL-6 was determined via qPCR (G) and ELISA $(\mathrm{H})$ respectively. Data are presented as mean \pm SEM from three independent, ${ }^{*} \mathrm{P}<0.05, \quad * * \mathrm{P}<0.01$.

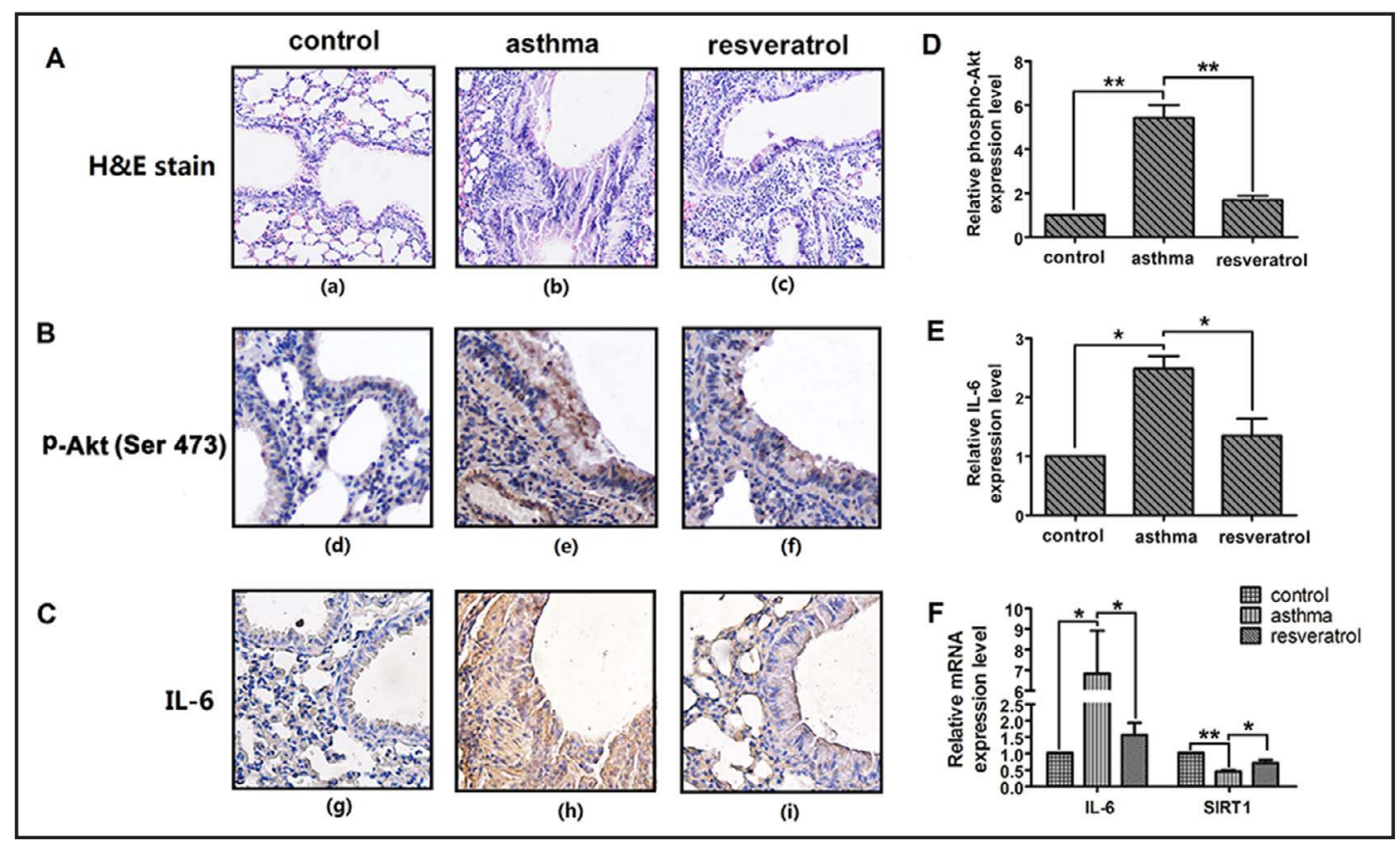

Fig. 6. SIRT1 activator inhibited IL-6 expression in vivo. Hematoxylin and eosin (H\&E) staining of bronchi (A), relative Akt phosphorylation at Ser473 (B) and IL-6 expression (C) in lung tissues were examined using immunohistochemical staining ( $\times 100)$. Relative Akt phosphorylation at Ser473 and IL-6 staining intensity was analyzed by measuring integrated optimal density (IOD) values, using Image-Pro Plus 6.0 (D, E). The mRNA expression of IL-6 and SIRT1 was determined via qPCR (F). The experiment used five mice in each group. Data are presented as mean $\pm \mathrm{SEM},{ }^{*} \mathrm{P}<0.05, \quad{ }^{*} \mathrm{P}<0.01$. 


\section{Cellular Physiology Cell Physiol Biochem 2017;43:1950-1960 \begin{tabular}{ll|l} 
and Biochemistry & $\begin{array}{l}\text { DOI 10.1159/000484119 } \\
\text { Published online: October 20, } 2017\end{array}$ & $\begin{array}{l}\text { O 2017 The Author(s). Published by S. Karger AG, Basel } \\
\text { www.karger.com/cpb }\end{array}$ \\
\cline { 2 - 3 }
\end{tabular} \\ Tang et al.: SIRT1 Increases IL-6 Expression by Activating Akt}

(Fig. 4E). Furthermore, using Akt siRNA, we observed that salermide-induced IL-6 protein expression was also attenuated (Fig. 4F). These results indicated that SIRT1 inhibition induced IL-6 expression in an Akt-dependent manner in human lung epithelial cells.

\section{SIRT1 activator inhibited IL-6 expression in human lung epithelial cells}

We then used resveratrol, a well-known SIRT1 activator [19] to investigate the effect of SIRT1 activation on IL-6 expression in human lung epithelial cells. As shown in Fig. 5A to D, resveratrol significantly inhibited SC79-induced relative Akt phosphorylation and IL-6 expression and salermide-induced IL-6 expression in 16HBE cells. Furthermore, we used RNA interference to knock down SIRT1 expression. We found silencing of SIRT1 increased Akt activation (Fig. 5E, F). In addition, resveratrol significantly inhibited IL-6 expression in 16HBE cells, however the effect of resveratrol on inhibiting IL-6 expression was attenuated after using SIRT1 RNAi (Fig. 5G, H). These results showed that SIRT1 activator such as resveratrol could be used to inhibit IL-6 expression in human lung epithelial cells.

\section{SIRT1 activator inhibited IL-6 expression in vivo}

It was reported SIRT1 activity was decreased in asthma patients, we then investigated the effect of SIRT1 activator (resveratrol) on regulating IL-6 expression in an asthma mouse model. Histopathologic examination of lung sections confirmed that airway inflammation was markedly attenuated in resveratrol-treated mice compared to OVA alone (Fig. 6A). Moreover, resveratrol increased SIRT1 expression but decreased relative Akt phosphorylation and IL-6 expression (Fig. 6B-F). Our results indicated that activator of SIRT1 could inhibit IL-6 expression in a mouse asthma model.

\section{Discussion}

Seven sirtuins (SIRT1-7) have been found in humans. Recently Colley et al. examined the level of expression of sirtuins (SIRT1, SIRT2, SIRT3, SIRT6, and SIRT7) in asthma patients, among which only SIRT1 protein showed any significant reduction in PBMCs from patients with asthma. This reduction of SIRT1 was also associated with overproduction of IL-4 [8]. The airway epithelium is a major contributor to asthma pathology and has been shown to produce an excess of inflammatory cytokines such as IL-6 [20]. However, the roles of SIRT1 in airway epithelial cells are still not fully understood. We herein further observed that inhibition of SIRT1 signaling by SIRT1 inhibitor or gene-silencing induced expression of IL- 6 in human lung epithelial cells. Conversely, activation of SIRT1 inhibited IL-6 expression in an asthmatic mouse model. Our study suggests new roles of SIRT1 in regulating inflammation in airway epithelial cells during the pathogenesis of asthma.

It has been reported that IL-6 expression is stimulated by transcription factor NF-kB, whose activity is regulated by Akt through phosphorylation of IкB kinases [21]. Enhanced

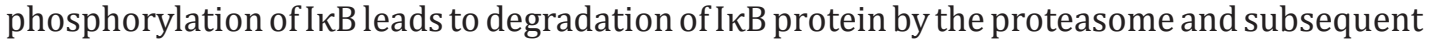
release of NF- $\kappa B$ to translocate to the nucleus to activate IL-6 expression [22]. Accordingly, we found that inhibition of SIRT1 by salermide was accompanied by increased AKT and IKB phosphorylation in 16HBE cells. We also found that the AKT inhibitor LY294002 or NF- $\kappa B$ inhibitor BAY11-7082 significantly inhibited salermide-induced IL-6 expression in 16HBE cells. Our results indicated that SIRT1 regulated IL-6 expression in an AKT/NF- $\mathrm{kB}$-dependent manner in airway epithelial cells.

The phosphatidylinositol 3-kinase/Akt pathway plays an important role in the signalling of cytokines and immune responses of eosinophils, T and B lymphocytes in asthma [23]. Increased Akt activity is found in eosinophils [24] derived from allergic asthmatics or lung tissue of asthmatic mice [25] and often correlates with enhanced allergic inflammatory reactions. Several studies have explored the mechanism underlying excessive Akt activation during asthma. For example, Li et al. found that TLR2 was associated with Akt activation [26]. In their cases, TLR2-knockout asthmatic mice had decreased Akt phosphorylation levels 


\section{Cellular Physiology Cell Physiol Biochem 2017;43:1950-1960

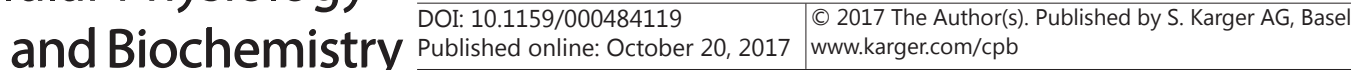 \\ Tang et al.: SIRT1 Increases IL- 6 Expression by Activating Akt}

compared to wild type asthmatic mice [26]. In line with their reports, we confirmed that the Akt phosphorylation level was significantly increased in asthma mice than in the control mice. Nevertheless, we found treatment of SIRT1 activator resveratrol displayed the decreased of Akt phosphorylation in vivo. Furthermore, we also found that SIRT1 suppression in airway epithelial cells increased Akt signalling activation. Our results extend previous observations that Akt phosphorylation is increased in asthma and point to inhibition of SIRT1 as one of the causes of the observed excessive Akt activation.

Dysregulation of SIRT1 is well documented in the pathogenesis of asthma. Some studies have suggested the stimuli for regulation SIRT1 expression in bronchial epithelial cells. It has been reported that exposure to cigarette smoke is an important stimulus for asthma and leads to more asthma symptoms [27]. Pace et al. found that stimulation with cigarette smoke extracts decreases the activity and levels of SIRT1 in 16HBE cells [28]. On the other hand, Caito et al. showed that excessive oxidative stress decreased SIRT1 enzymatic activity and marked the protein for proteasomal degradation [29]. However, whether these stimuli resulted in loss of SIRT1 function in the pathogenesis of asthma is still not known. We and other groups had demonstrated that SIRT1 played important roles in reduced inflammation and production of Th2 cytokines. Further investigation should be performed to elucidate the underlying mechanisms, which could be therapeutically useful for asthma treatment.

Asthma is a complex and heterogeneous chronic inflammatory disorder. The current therapy for asthma involves inhaled corticosteroids. It is effective, but many patients still remain poorly controlled. Several new treatments are in development such as targeting Treg cells [30], using kinase inhibitors [31] or using plant and herbal-derived compounds [32]. In this study, we found resveratrol, a type of polyphenol present in several plants, significantly inhibited IL-6 production in 16HBE cells. These results are consistent with previous reports of airway epithelial cells [33]. The effect of resveratrol on inhibiting IL-6 expression was attenuated after exposure to SIRT1 RNAi, which indicated that resveratrol regulated IL-6 expression through a SIRT1-dependent mechanism.We also found resveratrol treatment reduce IL- 6 expression levels in asthmatic mice. Our results indicated that resveratrol could be a promising anti-IL-6 treatment for asthma.

Taken together, our results indicated the potential role of SIRT1 in regulating inflammation by enhancing IL-6 expression during allergic asthma. Future work to understand the molecular mechanisms of SIRT1-mediated inflammatory response would provide more information for the development of novel therapeutic targets in asthma.

\section{Acknowledgements}

This work was supported by the National Natural Science Foundation of China (No.81402988), the Putuo key clinical specialist construction Programs (2014-A-23) and the Peiying Program of Putuo Hospital (2016206A ).

\section{Disclosure Statement}

All authors declared no conflicts of interest.

\section{References}

1 National Asthma E, Prevention P: Expert Panel Report 3 (EPR-3): Guidelines for the Diagnosis and Management of Asthma-Summary Report 2007. J Allergy Clin Immunol 2007;120:S94-138.

-2 Yoshizaki T, Schenk S, Imamura T, Babendure JL, Sonoda N, Bae EJ, Oh DY, Lu M, Milne JC, Westphal C, Bandyopadhyay G, Olefsky JM: SIRT1 inhibits inflammatory pathways in macrophages and modulates insulin sensitivity. Am J Physiol Endocrinol Metab 2010;298:E419-428. 


\section{Cellular Physiology Cell Physiol Biochem 2017;43:1950-1960 \begin{tabular}{l|l|l} 
and Biochemistry & Dublished online: October 20, 2017 & $\begin{array}{l}\text { C) } 2017 \text { The Author(s). Published by S. Karger AG, Basel } \\
\text { www.karger.com/cpb }\end{array}$ \\
\hline
\end{tabular}}

3 Lin Z, Fang D: The Roles of SIRT1 in Cancer. Genes Cancer 2013;4:97-104.

4 Rahman S, Islam R: Mammalian Sirt1: insights on its biological functions. Cell Commun Signal 2011;9:11.

5 Ichikawa T, Hayashi R, Suzuki K, Imanishi S, Kambara K, Okazawa S, Inomata M, Yamada T, Yamazaki Y, Koshimizu Y, Miwa T, Matsui S, Usui I, Urakaze M, Matsuya Y, Sasahara M, Tobe K: Sirtuin 1 activator SRT1720 suppresses inflammation in an ovalbumin-induced mouse model of asthma. Respirology 2013;18:332-339.

-6 Chen X, Lu Y, Zhang Z, Wang J, Yang H, Liu G: Intercellular interplay between Sirt1 signalling and cell metabolism in immune cell biology. Immunology 2015;145:455-467.

7 Wang Y, Li D, Ma G, Li W, Wu J, Lai T, Huang D, Zhao X, Lv Q Chen M, Wu B: Increases in peripheral SIRT1: a new biological characteristic of asthma. Respirology 2015;20:1066-1072.

-8 Colley T, Mercado N, Kunori Y, Brightling C, Bhavsar PK, Barnes PJ, Ito K: Defective sirtuin-1 increases IL-4 expression through acetylation of GATA-3 in patients with severe asthma. J Allergy Clin Immunol 2016;137:1595-1597 e1597.

-9 Chang SS, Vaz Fragoso CA, Van Ness PH, Fried LP, Tinetti ME: Association Between Combined Interleukin-6 and C-Reactive Protein Levels and Pulmonary Function in Older Women: Results from the Women's Health and Aging Studies I and II. J Am Geriatr Soc 2011;59:113-119.

10 Neveu WA, Allard JL, Raymond DM, Bourassa LM, Burns SM, Bunn JY, Irvin CG, Kaminsky DA, Rincon M: Elevation of IL-6 in the allergic asthmatic airway is independent of inflammation but associates with loss of central airway function. Respir Res 2010;11:28.

11 Camporeale A, Poli V: IL-6, IL-17 and STAT3: a holy trinity in auto-immunity? Front Biosci (Landmark Ed) 2012;17:2306-2326.

-12 Ma X, Reynolds SL, Baker BJ, Li X, Benveniste EN, Qin H: IL-17 Enhancement of the IL-6 Signaling Cascade in Astrocytes. J Immunol 2010;184:4898-4906.

13 Lin YL, Chen SH, Wang JY: Critical role of IL-6 in dendritic cell-induced allergic inflammation of asthma. J Mol Med (Berl) 2016;94:51-59.

14 Ni ZH, Tang JH, Chen G, Lai YM, Chen QG, Li Z, Yang W, Luo XM, Wang XB: Resveratrol inhibits mucus overproduction and MUC5AC expression in a murine model of asthma. Mol Med Rep 2016;13:287-294.

15 Lee JT, Gu W: SIRT1: Regulator of p53 Deacetylation. Genes Cancer 2013;4:112-117.

16 Wang Q, Zhang B, Yu JL: Farrerol inhibits IL-6 and IL-8 production in LPS-stimulated human gingival fibroblasts by suppressing PI3K/AKT/NF-kappaB signaling pathway. Arch Oral Biol 2016;62:28-32.

17 Yu H, Jiang W, Du H, Xing Y, Bai G, Zhang Y, Li Y, Jiang H, Zhang Y, Wang J, Wang P, Bai X: Involvement of the Akt/NF-kappaB pathways in the HTNV-mediated increase of IL-6, CCL5, ICAM-1, and VCAM-1 in HUVECs. PLoS One 2014;9:e93810.

18 Jo H, Mondal S, Tan D, Nagata E, Takizawa S, Sharma AK, Hou Q, Shanmugasundaram K, Prasad A, Tung JK, Tejeda AO, Man H, Rigby AC, Luo HR: Small molecule-induced cytosolic activation of protein kinase Akt rescues ischemia-elicited neuronal death. Proc Natl Acad Sci U S A 2012;109:10581-10586.

$>19$ Borra MT, Smith BC, Denu JM: Mechanism of human SIRT1 activation by resveratrol. J Biol Chem 2005;280:17187-17195.

-20 Martinez-Nunez RT, Bondanese VP, Louafi F, Francisco-Garcia AS, Rupani H, Bedke N, Holgate S, Howarth PH, Davies DE, Sanchez-Elsner T: A microRNA network dysregulated in asthma controls IL-6 production in bronchial epithelial cells. PLoS One 2014;9:e111659.

-21 Malanga D, De Marco C, Guerriero I, Colelli F, Rinaldo N, Scrima M, Mirante T, De Vitis C, Zoppoli P, Ceccarelli M, Riccardi M, Ravo M, Weisz A, Federico A, Franco R, Rocco G, Mancini R, Rizzuto A, Gulletta E, Ciliberto G, Viglietto G: The Akt1/IL-6/STAT3 pathway regulates growth of lung tumor initiating cells. Oncotarget 2015;6:42667-42686.

22 Tak PP, Firestein GS: NF-kappaB: a key role in inflammatory diseases. J Clin Invest 2001;107:7-11.

23 Cheng C, Ho WE, Goh FY, Guan SP, Kong LR, Lai WQ, Leung BP, Wong WS: Anti-malarial drug artesunate attenuates experimental allergic asthma via inhibition of the phosphoinositide 3-kinase/Akt pathway. PLoS One 2011;6:e20932.

24 Bracke M, van de Graaf E, Lammers JW, Coffer PJ, Koenderman L: In vivo priming of FcalphaR functioning on eosinophils of allergic asthmatics. J Leukoc Biol 2000;68:655-661.

25 Duan W, Aguinaldo Datiles AM, Leung BP, Vlahos CJ, Wong WS: An anti-inflammatory role for a phosphoinositide 3-kinase inhibitor LY294002 in a mouse asthma model. Int Immunopharmacol 2005;5:495-502. 


\section{Cellular Physiology Cell Physiol Biochem 2017;43:1950-1960

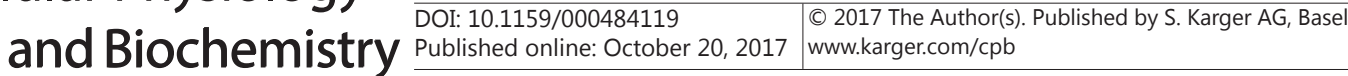 \\ Tang et al.: SIRT1 Increases IL-6 Expression by Activating Akt}

-26 Li X, Chen Q, Chu C, You H, Jin M, Zhao X, Zhu X, Zhou W, Ji W: Ovalbumin-induced experimental allergic asthma is Toll-like receptor 2 dependent. Allergy Asthma Proc 2014;35:e15-20.

27 Sathish V, Freeman MR, Long E, Thompson MA, Pabelick CM, Prakash YS: Cigarette Smoke and Estrogen Signaling in Human Airway Smooth Muscle. Cell Physiol Biochem 2015;36:1101-1115.

28 Pace E, Di Vincenzo S, Ferraro M, Bruno A, Dino P, Bonsignore MR, Battaglia S, Saibene F, Lanata L, Gjomarkaj M: Carbocysteine counteracts the effects of cigarette smoke on cell growth and on the SIRT1/ Fox03 axis in bronchial epithelial cells. Exp Gerontol 2016;81:119-128.

-29 Caito S, Rajendrasozhan S, Cook S, Chung S, Yao H, Friedman AE, Brookes PS, Rahman I: SIRT1 is a redoxsensitive deacetylase that is post-translationally modified by oxidants and carbonyl stress. FASEB J 2010;24:3145-3159.

30 Ding J, Su J, Zhang L, Ma J: Crocetin Activates Foxp3 Through TIPE2 in Asthma-Associated Treg Cells. Cell Physiol Biochem 2015;37:2425-2433.

-31 Song L, Tang H, Liu D, Song J, Wu Y, Qu S, Li Y: The Chronic and Short-Term Effects of Gefinitib on Airway Remodeling and Inflammation in a Mouse Model of Asthma. Cell Physiol Biochem 2016;38:194-206.

-32 Mali RG, Dhake AS: A review on herbal antiasthmatics. Orient Pharm Exp Med 2011;11:77-90.

33 Xie XH, Zang N, Li SM, Wang LJ, Deng Y, He Y, Yang XQ, Liu EM: Resveratrol Inhibits respiratory syncytial virus-induced IL-6 production, decreases viral replication, and downregulates TRIF expression in airway epithelial cells. Inflammation 2012;35:1392-1401. 